\title{
Exact solutions with AdS asymptotics of Einstein and Einstein-Maxwell gravity minimally coupled to a scalar field
}

\author{
Mariano Cadoni and Matteo Serra \\ Dipartimento di Fisica, \\ Università di Cagliari and INFN, \\ Sezione di Cagliari - Cittadella Universitaria, \\ 09042 Monserrato, Italy. \\ Salvatore Mignemi \\ Dipartimento di Matematica, \\ Università di Cagliari and INFN, \\ Sezione di Cagliari - viale Merello 92, \\ 09123 Cagliari, Italy. \\ (Dated: August 15, 2011)
}

\begin{abstract}
We propose a general method for solving exactly the static field equations of Einstein and EinsteinMaxwell gravity minimally coupled to a scalar field. Our method starts from an ansatz for the scalar field profile, and determines, together with the metric functions, the corresponding form of the scalar self-interaction potential. Using this method we prove a new no-hair theorem about the existence of hairy black-hole and black-brane solutions and derive broad classes of static solutions with radial symmetry of the theory, which may play an important role in applications of the AdS/CFT correspondence to condensed matter and strongly coupled QFTs. These solutions include: 1) fouror generic $(d+2)$-dimensional solutions with planar, spherical or hyperbolic horizon topology; 2) solutions with AdS, domain wall and Lifshitz asymptotics; 3) solutions interpolating between an AdS spacetime in the asymptotic region and a domain wall or conformal Lifshitz spacetime in the near-horizon region.
\end{abstract}

Contents

I. INTRODUCTION 2

II. A GENERAL METHOD FOR SOLVING THE FIELD EQUATIONS 4

III. DOMAIN WALLS AND SOLUTIONS CONFORMAL TO LIFSHITZ 6

A. Uncharged (Domain wall) solutions 6

B. Solutions conformal to Lifshitz spacetime

C. Alternative approach

IV. A NO-HAIR THEOREM 8

V. ASYMPTOTICALLY ADS SOLUTIONS

A. Uncharged solution

B. Charged solutions 13

C. Other solutions 14

VI. GENERALIZATION TO $d+2$ DIMENSIONS

A. Domain wall solutions

B. Charged solutions 16

C. Asymptotically AdS solutions 16

D. Other solutions

VII. SPHERICAL AND HYPERBOLIC SOLUTIONS 17

A. Uncharged black hole solutions 18 
B. Charged black hole solutions $\quad 18$

C. Asymptotically AdS solutions $\quad 18$

D. Charged asymptotically AdS solutions $\quad 19$

E. Spherical solutions generated from the planar ones $\quad 20$

\begin{tabular}{ll} 
F. Near-horizon, near-extremal solution with spherical topology & 20 \\
\hline
\end{tabular}

VIII. CONCLUSIONS

\begin{tabular}{ll} 
Acknowledgments & 22 \\
\hline
\end{tabular}

\begin{tabular}{ll} 
References & 22 \\
\hline
\end{tabular}

\section{INTRODUCTION}

In recent years there has been a renewed growing interest for the static black hole solutions of Einstein (and Einstein-Maxwell) gravity coupled to scalar fields, the search for these black holes and black branes being obviously focused on solutions dressed with a nontrivial profile of the scalar field (scalar hair).

In the past, the interest for these solutions was basically motivated either by the issue of the uniqueness of the Schwarzschild black hole and related no-hair theorems [1, 2] or by the quest for new black hole solutions in low-energy string models [3 -7].

The recent advances in string theory and in particular the AdS/CFT correspondence [8], have shifted the focus from the search of asymptotically flat to asymptotically anti-de Sitter (AdS) solutions. In this context, the applications of the AdS/CFT correspondence to holographic strongly coupled quantum field theories (QFTs) have generated a new interesting field of application for these solutions 9 14.

The shift to asymptotically AdS solutions permits to circumvent standard no-hair theorems, which relate the existence of black hole solutions with scalar hair to the violation of the positive energy theorem (PET) 15, 16]. Differently from the flat case, a scalar field in the AdS spacetime may have negative squared-mass $m^{2}$, without destabilizing the AdS vacuum, provided $m^{2}$ is above the Breitenlohner-Freedman (BF) bound [17].

On the other hand, static black hole and black brane solutions with non trivial scalar hair and AdS asymptotics play a crucial role in the holographic approach to strongly coupled QFTs based on the AdS/CFT correspondence. When the classical approximation for the bulk gravity theory is reliable, one can deal with strongly coupled QFTs at finite temperature in $d+1$ dimensions by investigating black holes in $d+2$ dimensions. In this context the nontrivial, coordinate-dependent scalar hair of the black hole solutions is interpreted either as a running coupling constant or as a scalar condensate in the dual QFT. In the first case the bulk scalar dynamics is very useful for holographic renormalization methods [18]. In the second case the scalar condensate is the origin of a rich phenomenology in the the dual QFT, reminiscent of well-known condensed matter systems 11 14, 19 22]. Following this line of reasoning, the existence of black hole solutions with scalar hair of Einstein gravity has become a central issue in several recent developments of the gravity/gauge field theory correspondence.

The best-known example is represented by the holographic superconductors. Below a critical temperature the bulk gravity theory, Einstein-Maxwell with a covariantly coupled scalar, allows for a black hole solutions with scalar hair [11, 14, 19, 21]. This corresponds to the formation of a charged condensate in the dual theory that breaks spontaneously a global $U(1)$ symmetry. The new phase is therefore characterized by phenomena typical of superfluid or superconducting systems. This basic structure has been generalized to a number of cases including, among others, Yang-Mill theories 23] and nonminimal couplings between the scalar and the electromagnetic (EM) field [20, 22, 24 28].

Another interesting feature of static black holes with scalar hair and AdS asymptotics, which is common to several models, is the presence in the near-horizon regime of Lifshitz-like solutions 
[20, 24, 25, 29]. These solutions represent a generalization of the Lifshitz spacetime, characterized by an anisotropic scaling isometry that may be relevant for quantum phase transitions [30 36], in presence of a scalar field.

Despite the growing importance played by static black hole solutions with scalar hair and AdS asymptotics, very little progress has been achieved for what concerns the derivation of exact analytical solutions. In fact, most of the usual methods used for finding exact, asymptotically flat, hairy solutions [3- 6] do not work in AdS spacetime. Most solutions of these kind, which have been derived and used in the literature, are numerical [11, 14, 19 22]. Very few exact analytical solutions are known: basically we have only the family of four-charge black holes in $\mathcal{N}=8$ fourdimensional gauged supergravity [37, 38], the solution with hyperbolic horizon of Ref. [39] and a few other examples some of them generated from asymptotically flat solutions [40 44].

Obviously, this situation has a negative impact on further developments of the subject. This is particularly true because the known no-hair theorems [15, 16] put loose constraints on the existence of black hole solutions with scalar hair. Therefore, they do not give stringent indications that can be used when searching for exact or numerical solutions of a given model.

Starting from these consideration, in this paper we first propose a general method for solving the field equations of Einstein and Einstein-Maxwell gravity minimally coupled to a scalar field $\phi$ in the static, radially symmetric, case. Our main idea is to reverse the usual method for solving the field equations. Usually, one determines the metric functions, the scalar field and the EM field, for a given form of the self-interaction potential $V(\phi)$. Instead of solving the field equations for a given potential, we will assume a given profile $\phi(r)$ for the scalar field and then we will solve the system for the metric functions and the potential.

This method is particularly suitable for application to the AdS/CFT correspondence. In this case the actual exact form of the potential $V(\phi)$ is not particularly relevant. What is more important is the behaviour of the scalar field, and in particular its fall-off behaviour at $r=\infty$.

We will apply our solving method to two different but related issues. First, we will apply it to find exact analytic solutions of Einstein and Einstein-Maxwell gravity with scalar hair. For the scalar field we use profiles which are very common for hairy black hole solutions in flat space, gauged supergravity, and Lifshitz spacetime, namely harmonic and logarithmic functions. This allows us to find exact solutions in several situations: four or generic $d+2$ spacetime dimensions, different topologies of the transverse space (planar, spherical, hyperbolic) and different asymptotics (anti-de Sitter, domain wall, conformal to Lifshitz spacetime). In particular we will derive exact solutions interpolating between an asymptotic AdS spacetime and a near-horizon domain wall or conformal Lifshitz spacetime. The models that we find contain as particular case the truncation to the abelian sector of $\mathcal{N}=8, D=4$ gauged supergravity.

Second, our method allows to write explicitly a formal solution of the field equations for an arbitrary potential. This fact will be used to prove a new no-hair theorem about the existence of black hole and black brane solutions of Einstein and Einstein-Maxwell gravity minimally coupled to a scalar field.

The structure of the paper is as follows. In Sect. I we present our general method for solving the field equations of Einstein-Maxwell-scalar gravity in $d+2$ dimension and planar, spherical or hyperbolic topology of the transverse sections. In Sect. III we apply this method to find domain wall and conformal Lifshitz black hole solutions in $d=2$ for the planar case. In Sect. IV we prove a new no-hair theorem for black hole solutions of Einstein-Maxwell gravity minimally coupled to a scalar field. In Sect. $\nabla$ we use our method to derive planar solutions with AdS asymptotics in $d=2$, both in the charged and uncharged case and discuss their near-horizon behaviour. The generalization of our solutions to the $d+2$-dimensional case and to spherical or hyperbolic solution is discussed, respectively in Sects. VI and VII] Finally in Sect. VIII we present our concluding remarks. 


\section{A GENERAL METHOD FOR SOLVING THE FIELD EQUATIONS}

In this paper we will consider Einstein-Maxwell gravity in $d+2$ dimensions (with $d \geq 2$ ), minimally coupled to a scalar field $\phi$, and with a generic self-interaction potential $V(\phi)$. The action is

$$
A=\int d^{d+2} x \sqrt{-g}\left(\mathcal{R}-2(\partial \phi)^{2}-F^{2}-V(\phi)\right) .
$$

The ensuing field equations take the form,

$$
\begin{aligned}
& \nabla_{\mu} F^{\mu \nu}=0 \\
& \nabla^{2} \phi=\frac{1}{4} \frac{d V(\phi)}{d \phi}, \\
& \mathcal{R}_{\mu \nu}-\frac{1}{2} g_{\mu \nu} \mathcal{R}=2\left(F_{\mu \rho} F_{\nu}^{\rho}-\frac{g_{\mu \nu}}{4} F^{\rho \sigma} F_{\rho \sigma}\right)+2\left(\partial_{\mu} \phi \partial_{\nu} \phi-\frac{g_{\mu \nu}}{2} \partial^{\rho} \phi \partial_{\rho} \phi\right)-\frac{g_{\mu \nu}}{2} V(\phi) .
\end{aligned}
$$

Throughout this paper we will investigate static solutions of the previous field equations exhibiting radial symmetry. Moreover, we will consider only purely electric solutions; magnetic solutions can be easily generated from the electric ones using the electro-magnetic duality. We adopt a Schwarzschild gauge to write the spacetime metric:

$$
d s^{2}=-U(r) d t^{2}+U^{-1}(r) d r^{2}+R^{2}(r) d \Omega_{(\varepsilon, d)}^{2},
$$

where $\varepsilon=0,1,-1$ denotes, respectively, the $d$-dimensional planar, spherical, or hyperbolic transverse space with metric $d \Omega_{(\varepsilon, d)}^{2}$. In these coordinates, the electric field satisfying (2.2) reads

$$
F_{t r}=\frac{Q}{R^{d}}
$$

with $Q$ the electric charge. With the parametrization (2.3), the field equations take the form

$$
\begin{aligned}
\frac{R^{\prime \prime}}{R} & =-\frac{2}{d}\left(\phi^{\prime}\right)^{2}, \quad\left(U R^{d} \phi^{\prime}\right)^{\prime}=\frac{1}{4} R^{d} \frac{d V}{d \phi} \\
\left(U R^{d}\right)^{\prime \prime} & =\varepsilon d(d-1) R^{d-2}+2 \frac{d-2}{d} \frac{Q^{2}}{R^{d}}-\frac{d+2}{d} R^{d} V, \\
\left(U R^{d-1} R^{\prime}\right)^{\prime} & =\varepsilon(d-1) R^{d-2}-\frac{2}{d} \frac{Q^{2}}{R^{d}}-\frac{1}{d} R^{d} V .
\end{aligned}
$$

We are mainly interested in black hole solutions of the field equations that are asymptotically AdS. Without loss of generality we can assume that $\phi \rightarrow 0$ as $r \rightarrow \infty$. Existence of the AdS vacuum therefore requires $V(0)<0$ and $V^{\prime}(0)=0$. Following a widespread convention we normalize $V(\phi)$ such that $V(0)=-d(d+1) / L^{2}$, where $L$ is the AdS length. Under this condition the simplest static black hole solution of the field equations is given by the Schwarzschild-AdS (SAdS) solution:

$$
U=\frac{r^{2}}{L^{2}}+\varepsilon-\frac{2 M}{r^{d-1}}, \quad R=r, \quad \phi=0, \quad Q=0 .
$$

Apart from SAdS and the $Q \neq 0$ Reissner-Nordstrom AdS black hole, the other solutions of (2.5), if they exist, will be characterized by a non-constant profile of the scalar field $\phi$. These solutions are very difficult to find, at least analytically. Eqs. (2.5) may be solved in closed form for some particular choice of the potential $V$, but for a generic potential there is no general solving method. Moreover, it is not completely clear if and when the field equations allow for regular black hole solutions. No-hair theorems relating the existence of black hole solutions to the violation of the positive energy theorem and to the breaking of the full AdS isometry group have been discussed in the literature [15, 16]. Explicit solutions, analytical or numerical, are known in a few cases. 
Nonetheless, a more precise statement about the existence of black hole solutions of the field equations (2.5) is still lacking.

In this paper we will often consider solutions with scalar hair and zero temperature (no horizon). With some abuse of terminology we will always call these solutions "extremal black hole solutions". Obviously, the use of this name is only strictly pertinent when the $T=0$ solution can be considered as the $T \rightarrow 0$ limit of black hole solutions with a regular horizon. As we will see in detail in the next sections, we will not be able to show that this is the case for all the hairy zero temperature solutions we will find. Nonetheless, we will use the word extremal black hole in the wide sense defined above.

Usually, one solves the field equations (2.5) by determining $U, R$, and $\phi$, for a given form of the potential $V(\phi)$. In this paper we will approach the problem in a reversed way. Instead of solving equations (2.5) for a given potential $V$, we will assume a given profile $\phi(r)$ for the scalar field and then solve the system for $U(r), R(r)$ and $V(\phi)$. Although at first sight this approach may seem rather weird, it is very useful for at least two reasons.

First, focusing on solutions with AdS asymptotics, in particular for what concerns application to the AdS/CFT correspondence, the actual exact form of the potential $V(\phi)$ is not particularly relevant. What is often more important is the behaviour of the scalar field $\phi(r)$, in particular its fall-off behaviour at $r=\infty$. In an asymptotically AdS spacetime the $r \rightarrow \infty$ behaviour of a scalar field is given by

$$
\phi \sim \frac{O_{-}}{r^{\Delta_{-}}}+\frac{O_{+}}{r^{\Delta_{+}}}, \quad \Delta_{ \pm}=\frac{(d+1) \pm \sqrt{(d+1)^{2}+4 m^{2} L^{2}}}{2},
$$

where $m^{2}$ is the mass of the scalar. Stability of the AdS vacuum requires $m^{2}$ to be above the BF bound, $m^{2} \geq-(d+1)^{2} /\left(4 L^{2}\right)$.

Moreover, in applications of the AdS/CFT correspondence to condensed matter physics, a nontrivial, $r$-dependent, profile of $\phi$ has a holographic interpretation in terms of a scalar condensate in the dual QFT triggering symmetry breaking and/or phase transitions [11, 14, 19 22]. If one is interested in reproducing phenomenological properties of strongly-coupled condensed matter systems, the actual form of the potential $V$ may be rather irrelevant. Conversely, it is the behavior of the scalar condensate that contains more physical information [11, 14, 19 22].

Second, our approach is very useful for setting up a new no-hair theorem about the existence of black hole solutions of the field equations. In fact, our method allows us to write explicitly a albeit formal - solution of the field equations for an arbitrary potential. This result will be used in Sect. IV to prove a new no-hair theorem about the existence of black hole solutions of minimally coupled Einstein-Maxwell-scalar gravity.

Our method for solving the field equations (2.5) works as follows. Assuming that the $r$ dependence of the scalar field $\phi(r)$ is given, and introducing the new variables $F, Y$ and $u$ defined as

$$
F(r)=-\frac{2}{d}\left(\phi^{\prime}\right)^{2}, \quad R=e^{\int Y}, \quad u=U R^{d},
$$

the field equations (2.5) become

$$
\begin{gathered}
Y^{\prime}+Y^{2}=F, \quad\left(u \phi^{\prime}\right)^{\prime}=\frac{1}{4} e^{d \int Y} \frac{d V}{d \phi}, \\
u^{\prime \prime}-(d+2)(u Y)^{\prime}=-2 \varepsilon(d-1) e^{(d-2) \int Y}+4 Q^{2} e^{-d \int Y}, \\
u^{\prime \prime}=\varepsilon d(d-1) e^{(d-2) \int Y}+2 \frac{d-2}{d} Q^{2} e^{-d \int Y}-\frac{d+2}{d} e^{d \int Y} V .
\end{gathered}
$$

The first equation in (2.9) is a first-order nonlinear equation for $Y$, known as the Riccati equation, which can be solved in a number of cases. Once the solution for $Y$ has been found we can integrate Eq. (2.10), which is linear in $u$, to obtain

$$
u=R^{d+2}\left[\int\left(4 Q^{2} \int \frac{1}{R^{d}}-2 \varepsilon(d-1) \int R^{d-2}-C_{1}\right) \frac{1}{R^{d+2}}+C_{2}\right],
$$


where $C_{1}$ and $C_{2}$ are integration constants. Finally, we can determine the potential $V(\phi)$ by using Eq. (2.11),

$$
V=\frac{d^{2}(d-1)}{d+2} \frac{\varepsilon}{R^{2}}+2 \frac{d-2}{d+2} \frac{Q^{2}}{R^{2 d}}-\frac{d}{d+2} \frac{u^{\prime \prime}}{R^{d}}
$$

while the metric functions read (cfr. 2.8)

$$
R=\Lambda e^{\int Y}, \quad U=\frac{u}{R^{d}},
$$

where we have introduced an integration constant $\Lambda$ coming from the integral of $Y$.

In the following sections we will use this method to find solutions of minimally coupled EinsteinMaxwell-scalar gravity in different spacetime dimensions and for planar, spherical, and hyperbolic topologies of the $d$-dimensional transverse section of the spacetime.

\section{DOMAIN WALLS AND SOLUTIONS CONFORMAL TO LIFSHITZ}

In this section, we consider the case of $(3+1)$-dimensional spacetime, i.e. $d=2$, and black brane solutions, i.e. $\varepsilon=0$. This is the most useful case for applications to holography. These solutions will be generalized to $d+2$ spacetime dimensions in section VI and to black holes with spherical $(\varepsilon=1)$ or hyperbolic $(\varepsilon=-1)$ symmetry in Sect. VII

Our method for solving the field equations (2.5) requires an ansatz for the scalar field. In this section we wish to find domain wall and Lifshitz-like solutions. Usually, these solutions appear when the scalar behaves as $\log r[20,22,24,25,27]$. The most natural ansatz is therefore:

$$
\gamma \phi=\log \frac{r}{r_{-}}
$$

where $\gamma$ and $r_{-}$are constants. Note that $r_{-}$has no particular physical meaning, but simply sets a length-scale.

\section{A. Uncharged (Domain wall) solutions}

Let us consider the solutions (2.12), (2.14) for $d=2, \varepsilon=0$. At first we examine the simplest case in which $Q=0$. Choosing $C_{1}=0$ and scaling the constants $C_{2}$ and $\Lambda$ to 1 , one gets

$$
U=R^{2}
$$

Hence, for this choice of the parameters, the solution takes the form of a domain wall,

$$
d s^{2}=U\left(-d t^{2}+d x^{2}+d y^{2}\right)+U^{-1} d r^{2} .
$$

Notice that in the relevant cases, even when $C_{1} \neq 0$ the corresponding term in Eq. (2.12) in the $r \rightarrow \infty$ limit is subleading, and therefore the solution is still asymptotical to a domain wall.

With the ansatz (3.1) the Riccati equation is solved by

$$
Y=\frac{\alpha}{r}, \quad \alpha(\alpha-1)=-\frac{1}{\gamma^{2}} .
$$

Parametrizing $\alpha$ and $\gamma$ as

$$
\gamma^{-1}=h \alpha=\frac{h}{h^{2}+1},
$$


the solution takes the form

$$
U=\left(\frac{r}{r_{-}}\right)^{\frac{2}{1+h^{2}}}-C_{1}\left(\frac{r}{r_{-}}\right)^{-\frac{1-h^{2}}{1+h^{2}}}, \quad R=\left(\frac{r}{r_{-}}\right)^{\frac{1}{1+h^{2}}}
$$

with potential

$$
V=-\frac{2\left(3-h^{2}\right)}{\left(1+h^{2}\right)^{2} r_{-}^{2}} e^{-2 h \phi}
$$

Hence the potential has a simple exponential form. If in the theory a length-scale $L$ is present, as happens for instance when the exponential potential arises as near-horizon approximation of an asymptotically AdS spacetime, one can trade $r_{-}$for $L$ using the invariance of the field equations under rescaling of $V \rightarrow \lambda V, U \rightarrow \lambda U$, yielding $V=-\left[\frac{2\left(3-h^{2}\right)}{\left(1+h^{2}\right) L^{2}}\right] e^{-2 h \phi}$.

In the extremal case, $C_{1}=0$, the solution (3.5) has the typical form of a single-scalar domain wall solution, $d s^{2}=(A r)^{\delta}\left(\eta_{\mu \nu} d x^{\mu} d x^{\nu}\right)+(A r)^{-\delta} d r^{2}$ [29, 45, 46]. These solutions preserve only the Poincaré isometry of the $3 \mathrm{D}$ transverse space and are related to solitonic supergravity domainwalls, resulting from various dimensional reductions of 10 and 11-dimensional maximal supergravity theories.

Moreover, domain wall solutions are conformal to AdS spacetime and have a consistent holographic interpretation, in terms of a dual QFT with only relativistic symmetry, for $\delta \geq 1$, which in our case implies $h^{2} \leq 1$.

One can easily calculate the curvature invariants for solution (3.5),

$$
\begin{aligned}
\mathcal{R} & =\frac{2}{\left(1+h^{2}\right)^{2} r_{-}^{2}}\left[3\left(h^{2}-2\right) x^{\frac{-2 h^{2}}{1+h^{2}}}-h^{2} \mu x^{\frac{-3-h^{2}}{1+h^{2}}}\right], \\
\mathcal{R}_{\mu \nu} \mathcal{R}^{\mu \nu} & =\frac{4}{\left(1+h^{2}\right)^{4} r_{-}^{4}}\left[\left(3 h^{4}-9 h^{2}+9\right) x^{\frac{-4 h^{2}}{1+h^{2}}}+3 h^{2}\left(1-h^{2}\right) \mu x^{-3}+h^{4} \mu^{2} x^{\frac{-6-2 h^{2}}{1+h^{2}}}\right],
\end{aligned}
$$

where $x=r / r_{-}$, showing that $r=0$ is a curvature singularity.

For $h^{2} \leq 3$ and $C_{1}>0$, our solution (3.5) represents a black brane with domain wall asymptotics, a singularity at $r=0$ and a horizon at $r_{h}=C_{1}^{\left(1+h^{2}\right) /\left(3-h^{2}\right)} r_{-}$. The horizon is regular and has negative curvature $\left(\mathcal{R}\left(r_{h}\right)<0, \mathcal{R}_{\mu \nu} \mathcal{R}^{\mu \nu}\left(r_{h}\right) \neq 0\right)$. The domain wall solution for $C_{1}=0$ display a naked singularity at the origin and may be seen as the $T=0$ extremal limit of (3.5). Notice however, that the solution at finite temperature breaks the Poincaré isometry of the extremal domain wall solution. For $h^{2}>3$, the solution is still valid, but its physical interpretation is less clear.

\section{B. Solutions conformal to Lifshitz spacetime}

Let us now consider the case of nonvanishing electric charge. In this case it is convenient to adopt the parametrization

$$
\alpha=\frac{h^{2}}{h^{2}+4}, \quad \gamma^{-1}=\frac{2 h}{h^{2}+4} .
$$

Using again the ansatz (3.1) and Eq. (3.3), the solution (2.12) reads

$$
\begin{aligned}
& U=\frac{Q^{2}}{\Lambda^{4}} \frac{\left(4+h^{2}\right)^{2} r_{-}^{2}}{\left(4-h^{2}\right)\left(2-h^{2}\right)}\left(\frac{r}{r_{-}}\right)^{2 \frac{4-h^{2}}{4+h^{2}}}\left[1-C_{1}\left(\frac{r}{r_{-}}\right)^{-\frac{4-h^{2}}{4+h^{2}}}+C_{2}\left(\frac{r}{r_{-}}\right)^{-4 \frac{2-h^{2}}{4+h^{2}}}\right], \\
& R=\Lambda\left(\frac{r}{r_{-}}\right)^{\frac{h^{2}}{4+h^{2}}},
\end{aligned}
$$


with potential

$$
V=-\frac{Q^{2}}{\Lambda^{4}}\left[\frac{4}{2-h^{2}} e^{-2 h \phi}+\frac{2 h^{2}\left(3 h^{2}-4\right)}{\left(4-h^{2}\right)\left(2-h^{2}\right)} C_{2} e^{-4 \phi / h}\right],
$$

where the integration constant $C_{1}$ and $C_{2}$ of (2.12) have been rescaled. The solutions hold for $h^{2} \neq 2,4$.

Setting $\Lambda^{2}=r_{-} Q$, the potential becomes independent from the electric charge. If an extra length scale $L$ is present, one can, like in the uncharged case, trade $r_{-}$for $L$ in the potential (3.9), so that it acquires a factor $1 / L^{2}$. The constant $C_{2}$ is a parameter of the action, that can be chosen to vanish. In such case, one is left with an exponential potential like in (3.6).

For $C_{2}=0$, the extremal $C_{1}=0$ case in Eq. (3.8) represents a solution which is conformal to the Lifshitz spacetime,

$$
d s^{2}=l^{2}\left(-\bar{r}^{2 z} d t^{2}+\frac{d \bar{r}^{2}}{\bar{r}^{2}}+\bar{r}^{2} d x^{i} d x^{i}\right)
$$

This can be easily shown by setting $\bar{r}=\left(r / r_{-}\right)^{-h^{2} /\left(4+h^{2}\right)}$ in Eq. (3.8). The metric (3.8) is conformal (with conformal factor $\bar{r}^{-4}$ ) to the Lifshitz metric (3.10) with $z=3-4 / h^{2}$ and $l=r_{-} \sqrt{\left(4-h^{2}\right)\left(2-h^{2}\right)} / h^{2}$. Obviously the anisotropic scaling transformation between space and time

$$
t \rightarrow \lambda^{z} t, \quad \bar{r} \rightarrow \lambda^{-1} \bar{r}, \quad x^{i} \rightarrow \lambda x^{i},
$$

which is an isometry of the Lifshitz metric (3.10), is not longer an isometry of our solution (3.8). However, its conformality with Lifshitz implies that it scales with a definite weight under the anisotropic scaling transformation (3.11): $d s^{2} \rightarrow \lambda^{4} d s^{2}$. In the remaining of this paper we will denote solutions which are conformal to Lifshitz spacetime simply as "conformal Lifshitz".

For $h^{2}<2, C_{2}=0$ and $C_{1}>0$ the solution represents a black brane with asymptotics conformal to Lifshitz, a singularity at $r=0$ and a regular horizon at $r_{h}=C_{1}^{\left(4+h^{2}\right) /\left(4-h^{2}\right)} r_{-}$. The conformal Lifshitz solution with $C_{1}=0$ may be seen as the $T=0$ extremal limit of (3.8). The solution at finite temperature do not follow the simple scaling behavior of the extremal solution.

If instead $C_{2} \neq 0$, two horizons may be present, depending on the value of the parameters. Moreover, the term in $C_{2}$ becomes dominant for $r \rightarrow \infty$ if $h^{2}>2$. In any case, the solutions (3.8) constitute a two-parameter family parametrized by $C_{1}$ and $Q$.

\section{Alternative approach}

When $C_{2}=0$, the solutions of this section can be obtained also by means of a more traditional approach, introduced in [3] and developed in several papers [4 [6, 47].

Parametrizing the metric and the electric field as

$$
d s^{2}=-e^{2 \nu} d t^{2}+e^{2 \nu+4 \rho} d \xi^{2}+e^{2 \rho}\left(d x^{2}+d y^{2}\right), \quad F_{t \xi}=e^{2 \nu} Q,
$$

where $\nu=\nu(\xi), \rho=\rho(\xi)$ and $\phi=\phi(\xi)$, one can in fact reduce the field equations to the form of a dynamical system, that admits a three-parameter family of regular black brane solutions. Exact solutions can be obtained in a special two-parameter case and coincide with those obtained above. The third parameter is presumably related to the scalar charge. More details will be given elsewhere [48].

\section{A NO-HAIR THEOREM}

In the previous section we have seen that minimally coupled Einstein-Maxwell-scalar gravity in $3+1$ dimensions allows for black brane solutions with either domain wall or conformal Lifshitz 
asymptotics. All these solutions correspond to an exponential potential $V(\phi)$ in the uncharged (domain wall) and charged (conformal Lifshitz) case, respectively. We will see later in Sects. VI and VII that these results hold also in $d+2$ dimensions and also for black holes, i.e. solutions with spherical horizons.

An important issue in this context is the question about the existence of regular, static black hole solutions of Einstein-scalar gravity with AdS asymptotics beyond the Schwarzschild and ReissnerNordstrom solutions, i.e. of solutions endowed with non trivial scalar hair.

This problem has been already discussed in the literature. In particular, it has been argued that necessary conditions for the existence of such black hole solutions are the violation of the PET and, in theories where the scalar field has negative local maxima, the breaking of the full AdS isometry group [15, 16]. These results rule out black hole solutions with scalar hair and positive squared-mass $m^{2}{ }^{1}$ but allows for the existence of these solutions when $m^{2}$ is negative but above the BF bound. Some examples of regular (analytical or numerical) AdS black hole solutions with scalar hair are known. An analytical solution has been obtained in Ref. [39], whereas numerical solutions have been found in the context of designer gravity [49], Einstein-Maxwell gravity with covariantly coupled scalar [1, 14, 19, 21] and Einstein-Maxwell dilaton gravity 20, 22].

We prove here, using the reformulation of the field equations discussed in Sect. III a new nohair theorem about the existence of regular hairy black hole solutions of Einstein-Maxwell-scalar gravity. We will consider for simplicity the $d=2$ case, but our theorem can be trivially generalized to arbitrary $d+2$ dimensions. We will first consider the uncharged and planar case $Q=\varepsilon=0$ and then we will generalize our argument to the charged and $\varepsilon= \pm 1$ cases.

A key ingredient for our argument is the existence of an extremal $T=0$ hairy black hole solution. We will prove the validity of the following three statements about black hole solutions of Einstein gravity minimally coupled to a scalar field:

1) One-parameter families of asymptotically AdS black brane solution with nontrivial scalar hair exist only if the field equations (2.5) admit an extremal $T=0, U=R^{2}$ solution.

2) Black-brane solutions that asymptotically approach the domain wall solution (3.5) exist in some range of the parameters for the case of an exponential potential $V(\phi)$.

3) The allowed asymptotically AdS hairy black brane solutions necessarily have a scalar hair that depends on the black brane temperature $T$. Solutions with temperature-independent scalar hair exist only for the case of domain wall spacetimes (3.5).

In order to prove part 1) of the theorem we start from equation (2.12), set $d=2, \varepsilon=0$ and fix the physically irrelevant integration constant $C_{2}=1$. We get the general form of the solution of the field equation (2.5) for the metric function $U$

$$
U=R^{2}\left(1-C_{1} \int \frac{1}{R^{4}}\right)
$$

The integration constant $C_{1}$ is determined in terms of the mass $M$ (or equivalently of the temperature $T$ ) of the solution.

Assuming the existence of a one-parameter family of black brane solutions with a regular horizon at $r=r_{h}$ we have to require $U\left(r_{h}\right)=0, R\left(r_{h}\right) \neq 0$. This implies that the horizon is determined by the equation

$$
1-C_{1} \int \frac{1}{R^{4}}=0
$$

whereas for $C_{1}=0$ we have an extremal $T=0$ domain wall solution with $U=R^{2}$.

Let us now assume that the field equations do not admit the $C_{1}=0$ extremal solution. Inserting Eq. (4.1) into the third field equation in (2.5), $C_{1}$ becomes completely determined in terms of the

1 As a consequence, asymptotically flat black holes are ruled out completely because the Minkowski vacuum would be unstable. 
functions $R$ and $\phi$. This, at least in principle, can be used to eliminate the integration constant $C_{1}$ from the field equations (2.5), which can now be used to determine the solutions for $R$ and $\phi$. As a consequence, the solutions for $R$ and $\phi$ will not depend on $C_{1}$, i.e. they will be temperature independent.

Let us now pick up a particular -albeit generic- solution of the field Eq. (2.5) with $C_{1}=C_{1}^{(0)}$, denote it with $\left(U_{0}, R_{0}, \phi_{0}\right)$ and decompose the general solution of the field equations (2.5) as follows:

$$
\left(U=U_{0}+\tilde{U}\left(C_{1}, r\right), \quad \phi, \quad R\right) .
$$

Because $R$ and $\phi$ do not depend on $C_{1}$, we must have $\phi=\phi_{0}$ and $R=R_{0}$. Substitution of Eq. (4.3) into Eq. (2.5) gives $\left(\tilde{U} R^{2}\right)^{\prime \prime}=0,\left(\tilde{U} R^{2} \phi^{\prime}\right)^{\prime}=0$, which implies $\phi=c \log \frac{r}{r_{0}}$, with $c, r_{0}$ integration constants. According to Eqs. (3.1)-(3.6), this is only possible for an exponential potential $V(\phi)$ and gives the domain wall solution (3.5). From this, part 1) and part 2) of the no-hair theorem follow immediately.

Obviously, if the field equation allow the $C_{1}=0$ solution the previous derivation fails. We can choose in Eq. (4.3) $U_{0}$ as the $C_{1}=0$ solution, whereas $\phi$ and $R$ do not need to be independent from $C_{1}$.

Statement 3) can be proved with a slight modification of the previous argument. One begins by noticing that, owing to the first equation in (2.5), a temperature-independent scalar hair implies that also the function $R$ is temperature-independent. One then assumes the existence of a oneparameter family of black brane solutions of the field equations (2.5) $\left(U\left(C_{1}\right), \phi, R\right)$ with $\phi$ and $R$ independent of $C_{1}$. Repeating the argument starting from Eq. (4.3) one easily finds that the one-parameter family of hairy solutions $\left(U\left(C_{1}\right), \phi, R\right)$ exists only in the case of an exponential potential and is given by the black brane solutions (3.5).

The previous derivation can be easily extended to the charged case $Q \neq 0$ and to $\varepsilon= \pm 1$. The only new ingredient is that now the general solution of the field equations (2.5) is determined by

$$
U=R^{2}\left[1+\int\left(4 Q^{2} \int \frac{1}{R^{2}}-2 \varepsilon r-C_{1}\right) \frac{1}{R^{4}}\right],
$$

rather than by Eq. (4.1). Since for $Q \neq 0$ or $\varepsilon \neq 0$ the solution (4.4) with $C_{1}=0$ is no longer given by $U=R^{2}$, in general, it will not necessarily be an extremal $T=0$ solution. This is related to the fact that $C_{1}$ will now be determined not only in terms of the black hole mass $M$, but in terms of $Q$ as well. As a consequence, the spacetime will in general have an inner and outer horizon. Statement 1) then holds in a much weaker form: One-parameter families of asymptotically charged AdS black hole solution with nontrivial scalar hair exist only if the field equations (2.5) admit a black hole solution with $C_{1}=0$. Because the $C_{1}=0$ solution is not necessarily extremal this statement is not particularly useful.

On the other hand statements 2) and 3) do not depend on the existence of an extremal solution. Their generalization to the charged and $\varepsilon= \pm 1$ case is almost trivial. Statement 2) now affirms that charged black brane/black hole solutions that asymptotically approach the conformal Lifshitz spacetime (3.8) exist, in some range of the parameters, for the case of an exponential potential $V(\phi)$. Statement 3) still remains true in the form given above also for the case of charged and $\varepsilon= \pm 1$ black holes.

Concerning statement 3) it is important to stress that this theorem does not apply to the case of a nonminimal coupling between the scalar and the gauge field. In the latter case we have an additional term depending on the derivative of the coupling function between the scalar and $F^{2}$ in the last equation of (2.5). The effect of this term is to allow for solutions with two integration constants $r_{ \pm}$, with $Q \sim r_{-} r_{+}, T \sim\left(r_{+}-r_{-}\right)$, whereas the scalar field depends on $r_{-}$only. Hence the scalar hair is independent from the black hole temperature but is related to $Q$. This result is perfectly consistent with the well-established existence of black holes with temperature-independent scalar charges in models with a non-minimally coupled scalar field.

We conclude this section by listing the classes of static black hole solutions of Einstein-Maxwellscalar gravity that may exist in view of the above no-hair theorems. 
- Models with an exponential potential admit, at least in some range of the parameters, a one-parameter family of domain wall $(Q=0, \varepsilon=0)$, conformal Lifshitz $(Q \neq 0, \varepsilon=0)$, black brane or black hole $(Q=0$ or $Q \neq 0$ and $\varepsilon= \pm 1)$ solutions.

- The existence of asymptotically AdS uncharged black brane solutions with scalar hair is tightly constrained. Apart from the violation of the PET, a further necessary, but not sufficient, condition for their existence is that the field equations allow for an extremal $T=0$ solution.

- The existence of hairy asymptotically AdS charged black branes, or charged and uncharged black holes, is very loosely constrained by the above no-hair theorem.

- For all cases (charged and uncharged, black branes and black holes) the allowed hairy AdS solutions must have a temperature-dependent scalar hair.

\section{ASYMPTOTICALLY ADS SOLUTIONS}

In this section we will derive asymptotically AdS solutions with scalar hair of the field equations (2.5) for $d=2$ and $\varepsilon=0$. As explained in Sect. [II] in order to have asymptotically AdS solution we require the potential to satisfy, $V(0)<0, V^{\prime}(0)=0$ and we normalize $V$ using $V(0)=-6 / L^{2}$.

As usual, the starting point of our solving method is an ansatz for the scalar field. Inspired by known solutions in flat spacetime and in gauged supergravity [3 6, 37], we use an ansatz in which $\phi$ is expressed in terms of a four-dimensional harmonic function $X$,

$$
\gamma \phi=\log X, \quad X=1-\frac{r_{-}}{r},
$$

where $\gamma$ and $r_{-}$are constants. Notice that with this ansatz, in the asymptotical AdS region the scalar field $\phi$ is a tachyonic excitation with mass above the BF bound in $4 \mathrm{D}, m^{2}=-2 / L^{2}$. Expanding Eq. (5.1) near $r=\infty$ and comparing with Eq. (2.7) one finds that the asymptotic behaviour of the scalar field is characterized by $\Delta_{ \pm}=2,1$ and by $O_{+}=r_{-} O_{-} / 2$. This tells us that we are dealing with so called designer gravity models [49].

Given the ansatz (5.1) the Riccati equation (2.9) can be solved in terms of the harmonic function $X$ to give,

$$
R=\Lambda r X^{\beta+\frac{1}{2}}, \quad \beta^{2}-\frac{1}{4}=-\frac{1}{\gamma^{2}} .
$$

where $\Lambda$ can be set to 1 without loss of generality, if $\varepsilon=0$ and $Q=0$. Notice that the previous equation implies

$$
-\frac{1}{2}<\beta<\frac{1}{2} .
$$

As usual we will proceed by discussing separately uncharged and charged solutions.

\section{A. Uncharged solution}

Let us set $Q=0$, in Eq. (2.12) and first consider the $C_{1}=0$ extremal solutions. The constant

$C_{2}$ essentially determines the normalization of the potential. This is fixed by choosing $C_{2}=1 / L^{2}$. With these assumptions, Eq. (2.12) and (2.13) give, respectively, the solution for the metric and the scalar potential

$$
\begin{aligned}
U & =R^{2}=\frac{r^{2}}{L^{2}}\left(1-\frac{r_{-}}{r}\right)^{2 \beta+1}, \\
V_{1}(\gamma, \phi) & =-\frac{2}{L^{2}} e^{2 \gamma \beta \phi}\left[2-8 \beta^{2}+\left(1+8 \beta^{2}\right) \cosh (\gamma \phi)-6 \beta \sinh (\gamma \phi)\right] .
\end{aligned}
$$


One can easily check that these solutions represent domain walls with $\mathrm{AdS}_{4}$ asymptotics. Calculating the periodicity of the 2D Euclidean section one can also check that the solution is an extremal $T=0$ solution.

The potential (5.5) interpolates smoothly between the asymptotic AdS region at $\phi=0$ and a $\phi \rightarrow \infty$ region (a near-horizon region) where the potential behaves exponentially,

$$
V(\phi)=-\frac{(2 \beta+1)(4 \beta+1)}{L^{2}} e^{\gamma(2 \beta-1) \phi} .
$$

Moreover, it contains as a special case, $\beta=0$, the potential resulting from truncation to the abelian sector of $\mathcal{N}=8, D=4$ gauged supergravity [49],

$$
V(\phi)=-\frac{2}{L^{2}}(\cosh 2 \phi+2)
$$

In this case the solution (5.4) takes the particularly simple form

$$
U=R^{2}=\frac{r^{2}}{L^{2}}-\frac{r r_{-}}{L^{2}}, \phi=\frac{1}{2} \log \left(1-\frac{r_{-}}{r}\right) .
$$

The model described by the potential (5.5) becomes very simple also for $\beta=1 / 4$,

$$
V(\phi)=-\frac{6}{L^{2}} \cosh \frac{2 \phi}{\sqrt{3}} .
$$

The potential (5.5) remains invariant under the duality transformation

$$
\phi \rightarrow-\phi, \quad \beta \rightarrow-\beta
$$

This symmetry of the action can be used to generate a new dual solution from Eq. (5.4),

$$
U=R^{2}=\frac{r^{2}}{L^{2}} X^{-2 \beta+1}, \quad \gamma \phi=-\log X
$$

Notice that for the supergravity model (5.7) the symmetry transformation is simply $\phi \rightarrow-\phi$, whereas solution (5.8) becomes self-dual.

Because the model with potential (5.5) admits the $C_{1}=0$ solution, statement 1) of the no-hair theorem discussed in the previous section implies that also non extremal black brane solutions with $C_{1} \neq 0$ can in principle exist. Unfortunately, our method does not allow to find such a solutions. Naively, one could think that these solutions can be derived just by using Eq. (5.2) into Eq. (2.12) with $C_{1} \neq 0$. This is not the case not only because the resulting potential $\tilde{V}$ is different from (5.5) but, more importantly, because $\tilde{V}$ will depend explicitly on $C_{1}$, which instead should be a free integration constant related to the mass of the solution.

Notice that a one-parameter family of solutions can be generated from Eq. (2.12) with $C_{1} \neq 0$ by using the invariance of the field equations under the rescaling $R \rightarrow \lambda R$, to let the potential depend only on the ratio $C_{2} / C_{1}$, whereas $U$ depends on both $C_{2}$ and $C_{1}$. However, in this case the solution $C_{1}=0$ is not allowed. Hence the no-hair theorem of the previous section implies that this family of solutions are not black branes. Thus non-extremal black brane solutions of models with the potential (5.5), if they exist, have to be found numerically.

The hairy extremal solution (5.4) interpolates between an AdS vacuum at $r=\infty$ and a domain wall solution (3.5) near $r=r_{-}$. This can be easily shown by expressing solution (5.4) in the near-horizon approximation $r \sim r_{-}$. Shifting $r \rightarrow r+r_{-}$and expanding near $r=0$ one finds at leading order,

$$
\gamma \phi=\ln \frac{r}{r_{-}}, \quad U=R^{2}=A^{2}\left(\frac{r}{r_{-}}\right)^{2 \beta+1}, \quad A=\frac{r_{-}}{L}
$$


As expected, this solution is easily recognized, just by setting $\beta=\left(1-h^{2}\right) /\left(2+2 h^{2}\right)$ and by rescaling $V$ (in the way explained after Eq. (3.5)) as the exact solutions (3.5) with $C_{1}=0$ of a model with near-horizon exponential potential (5.6).

The near-extremal solutions with a horizon, corresponding to solutions (3.5) with $C_{1} \neq 0$ are given by

$$
U=A^{2}\left(\frac{r}{r_{-}}\right)^{2 \beta+1}-\mu\left(\frac{r}{r_{-}}\right)^{-2 \beta},
$$

whereas $\phi$ and $R$ are given as in Eq. (5.12). We stress again that Eq. (5.12) and (5.13) are exact solution of the near-horizon approximate form of the potential (5.6) but only leading-order solution of the near-horizon approximation for the exact potential (5.5).

The near-horizon, extremal and near-extremal solution, corresponding to the dual solution (5.11) can be easily obtained from Eq. (5.13) just by using the duality transformation (5.10).

\section{B. Charged solutions}

Following the same steps described in the previous subsection we now derive $Q \neq 0$ hairy black brane solutions of the field equations (2.5) with AdS asymptotics.

The $C_{1}=C_{2}=0$ solution, corresponding to the ansatz (5.1) for the scalar, is obtained by substituting the solution (5.2) of the Riccati equation into Eq. (2.12),

$$
\begin{aligned}
U & =\frac{r^{2}}{L^{2}}\left(1-\frac{r_{-}}{r}\right)^{-2 \beta}\left(1-\frac{r_{1}}{r}\right)\left(1-\frac{r_{2}}{r}\right), \\
R & =\Lambda \frac{r}{r_{-}}\left(1-\frac{r_{-}}{r}\right)^{\beta+\frac{1}{2}}, \quad \gamma \phi=\log \left(1-\frac{r_{-}}{r}\right), \quad \frac{1}{4}-\beta^{2}=\gamma^{-2},
\end{aligned}
$$

where $\Lambda^{2}=Q L /\left(|\beta| \sqrt{\frac{3}{2}\left(36 \beta^{2}-1\right.}\right), r_{1,2}=\left(r_{-} / 2\right)\left(6 \beta+1 \pm \sqrt{36 \beta^{2}-1}\right)$ and $1 / 6<|\beta|<1 / 2$.

Using Eq. (2.13), the corresponding potential turns out to be

$$
\begin{aligned}
V_{2}(\gamma, \phi) & =-\frac{2}{L^{2}} e^{-4 \gamma \beta \phi}\left(4 \beta^{2}-1\right)\left[-\left(36 \beta^{2}+1\right) \cosh (\gamma \phi)+27 \beta^{2}-2-12 \beta \sinh (\gamma \phi)\right] \\
& +3 \beta^{2}\left(1+12 \beta^{2}\right) \cosh (2 \gamma \phi)+24 \beta^{3} \sinh (2 \gamma \phi) .
\end{aligned}
$$

Eqs. (5.14) represent a one-parameter (the charge) family of asymptotically AdS solutions of the model (5.15) for a fixed value of the mass (or temperature). As pointed out in Sect. IV] in the charged case the $C_{1}=0$ solution does not necessarily correspond to extremal $T=0$ black brane solutions. Moreover, in this case statement 1) of the no-hair theorem of the previous section is not useful for guessing about the existence of a full two-parameter (charge $Q$ and mass $M$ ) family of hairy black brane solutions.

The potential (5.15) is invariant under the duality symmetry (5.10). The dual solutions are easily obtained using Eq. (5.10) into Eq. (5.14).

The geometrical and thermal properties of the solution (5.14) depend on the value of $\beta$. In the parameter region $-1 / 2<\beta<-1 / 6$ we have $r_{1}, r_{2}<r_{-}$. Because $r_{-}$is the origin of the radial coordinate $r$, there are no horizons and the solution is an extremal $T=0$ solution. For $1 / 6<\beta<1 / 2$ we get $r_{1}>r_{-}$and we have an horizon. Because $r_{1}$ is just a simple (not double) root of $U$, the solution does not represent an extremal $T=0$ solution.

Solution (5.14) for $-1 / 2<\beta<-1 / 6$ interpolates between an AdS vacuum at $r=\infty$ and a conformal Lifshitz solution (3.8) with $C_{1}=C_{2}=0$ in the near-horizon limit $r \sim r_{-}$. In fact, shifting $r \rightarrow r+r_{-}$and expanding near $r=0$, Eq. (5.14) becomes

$$
\gamma \phi=\ln \frac{r}{r_{-}}, \quad U=B\left(\frac{r}{r_{-}}\right)^{-4 \beta}, \quad R=\frac{r_{-}}{L}\left(\frac{r}{r_{-}}\right)^{\beta+\frac{1}{2}}
$$


where $B$ is a constant depending on $\beta$ and $r_{-}$. Solution (5.16) has the conformal Lifshitz form (3.8). On the other hand for $1 / 6<\beta<1 / 2$ we have $U \sim r$ and $R \sim$ const., which seems to indicate that in this case the solution has to interpreted as an extremal $T \neq 0$ solution.

Analogously to the $Q=0$ case, one can also write down near-extremal, approximate solutions with an horizon (black brane):

$$
\gamma \phi=\ln \frac{r}{r_{-}}, \quad U=B\left(\frac{r}{r_{-}}\right)^{-4 \beta}-\mu\left(\frac{r}{r_{-}}\right)^{-2 \beta}, \quad R=\frac{r_{-}}{L}\left(\frac{r}{r_{-}}\right)^{\beta+\frac{1}{2}}
$$

Also in the charged case, both the $C_{1}=0$ solution (5.16) and the near-extremal solution (5.17) are exact solution of an Einstein-Maxwell-scalar gravity model with an exponential potential given by the leading term in the $\phi \rightarrow \infty$ expansion of the potential (5.15).

\section{Other solutions}

In this subsection we present a further example of the use of our general method for generating exact solutions of (2.5) with AdS asymptotic behavior, for $Q=0, d=2$ and $\varepsilon=0$.

As ansatz for the scalar field we choose a combination of harmonic functions in $n+2$ dimensions $^{2}$

$$
\phi=\frac{\sqrt{2 n-1}}{2 n} \log \frac{X_{+}}{X_{-}}, \quad X_{ \pm}=1 \pm\left(\frac{r_{-}}{r}\right)^{n}, \quad n>\frac{1}{2} .
$$

In an asymptotically AdS spacetime, this corresponds to a scalar excitation near $\phi=0$ of mass

$$
m^{2}=-\frac{n(3-n)}{L^{2}} .
$$

The scalar excitation is a tachyon with mass above the BF bound for $1 / 2<n<3$. The PET implies the non existence of black brane solutions for $n \geq 3$.

The Riccati equation (2.9) is solved by

$$
Y=\frac{r^{2 n-1}}{r^{2 n}-r_{-}^{2 n}}
$$

In the uncharged case, $Q=0$, Eq. (2.12) with $C_{1}=0$ and $C_{2}=1 / L^{2}$ gives the solution

$$
U=R^{2}=\frac{r^{2}}{L^{2}}\left[1-\left(\frac{r_{-}}{r}\right)^{2 n}\right]^{\frac{1}{n}}, \quad \phi=\frac{\sqrt{2 n-1}}{2 n} \log \frac{r^{n}+r_{-}^{n}}{r^{n}-r_{-}^{n}},
$$

which represents an asymptotically AdS domain-wall solution. As expected also in this case the solution is a $T=0$ extremal solution. Eq. 2.13) gives the potential :

$$
V(\phi)=-\frac{2}{L^{2}}\left(\cosh \frac{a \phi}{2}\right)^{2-\frac{2}{n}}[(2-n) \cosh a \phi+(n+1)], \quad a=\frac{2 n}{\sqrt{2 n-1}} .
$$

Notice that this potential is invariant under the duality transformation $\phi \rightarrow-\phi$. The potential (5.22) smoothly interpolates between the asymptotical AdS region at $\phi=0$ and the $\phi \rightarrow \infty$ near-horizon region where the potential has the exponential behavior

$$
V(\phi)=-\frac{(2-n)}{L^{2}} 2^{\frac{2}{n}-2} e^{2 \sqrt{2 n-1} \phi} .
$$

\footnotetext{
${ }^{2}$ We do not limit ourselves to an integer $n$, but we take $n$ real.
} 
In the special case $n=2$ the potential takes a very simple form,

$$
V(\phi)=-\frac{6}{L^{2}} \cosh \frac{2 \phi}{\sqrt{3}} .
$$

Also for the case of the potential (5.22) hold the same considerations concerning the existence of non-extremal black brane solutions as those discussed in subsection VA for the potential (5.5).

The extremal solution (5.21) interpolates between an AdS vacuum at $r=\infty$ and a domain wall solution of the form (3.5) with $C_{1}=0$ near $r=r_{-}$.

This can be easily seen by working in the near-horizon approximation. Shifting $r \rightarrow r+r_{-}$and expanding near $r=0$, Eq. (5.21) becomes at leading order

$$
\phi=-\frac{1}{a} \ln \frac{r}{r_{-}}, \quad U=R^{2}=D^{2}\left(\frac{r}{r_{-}}\right)^{\frac{1}{n}}, \quad D=\frac{r_{-}}{L}(2 n)^{1 /(2 n)} .
$$

Near-extremal approximate solutions with a horizon have the form (3.5) with $C_{1} \neq 0$ and are given by

$$
U=D^{2}\left(\frac{r}{r_{-}}\right)^{1 / n}-\mu\left(\frac{r}{r_{-}}\right)^{1-1 / n}
$$

whereas $\phi$ and $R$ are given as in Eq. (5.25). As expected, solution (5.26) is an exact solution of a model with the exponential potential (5.23).

\section{GENERALIZATION TO $d+2$ DIMENSIONS}

In this section we generalize the black brane solutions found in the $d=2$ case to $d+2$ dimensions.

\section{A. Domain wall solutions}

We start again from the ansatz (3.1). When $d \neq 2$ the Riccati equation (2.9) is solved by

$$
Y=\frac{\alpha}{r}, \quad \alpha(\alpha-1)=-\frac{2}{d} \gamma^{-2} .
$$

It is useful to parametrize $\alpha$ and $\gamma$ as follows:

$$
\alpha=\frac{2}{2 d+h^{2}}, \quad \gamma^{-1}=\frac{d h}{2+d h^{2}}
$$

Redefining the constant $C_{2}$ in Eqs. (2.8) and (2.12), and rescaling $C_{1}$, the solution takes the form:

$$
\begin{aligned}
U & =\left(\frac{r}{r_{-}}\right)^{\frac{4}{2+d h^{2}}}-C_{1}\left(\frac{r}{r_{-}}\right)^{\frac{d h^{2}-2 d+2}{2+d h^{2}}}, \\
R & =\left(\frac{r}{r_{-}}\right)^{\frac{2}{2+d h^{2}}}, \\
V & =-\frac{2 d\left[2(d+1)-d h^{2}\right]}{\left(2+d h^{2}\right)^{2} r_{-}^{2}} e^{-2 h \phi},
\end{aligned}
$$

As usual, the $r_{-}$in the potential can be substituted by the AdS scale $L$, using the invariance of the field equations under rescaling of $V$ and $U$.

As in $d=2$, choosing $C_{1}=0$ we obtain the typical domain wall solution $U=R^{2}$. For $h^{2} \leq 2 / d$, the domain wall solution (6.3) with $C_{1}=0$ has a consistent holographic interpretation and a singularity at $r=0$. For $C_{1} \geq 0$ and $h^{2} \leq 2+2 / d$, the solution (6.3) is asymptotical to the domain wall solution, and has a horizon at $r_{h}=C_{1}^{\left(2+d h^{2}\right) /\left(2 d+2-d h^{2}\right)} r_{-}$. 


\section{B. Charged solutions}

The previous solution can be generalized to the case $Q \neq 0$. This is the only charged solution, among those found for $d=2$, that can be computed in closed form in $d+2$ dimensions.

The ansatz for the scalar field is still given by 3.1), whereas the solution for the Riccati equation is the same as in Eq. (6.1). In the case at hand, it is convenient to choose the following parametrization for $\alpha$ and $\gamma$ :

$$
\alpha=\frac{h^{2}}{2 d+h^{2}}, \quad \gamma^{-1}=\frac{d h}{2 d+h^{2}} .
$$

Equations (2.12), (6.1) and (2.13) give (after a rescaling of the integration constant $C_{1}$ and $C_{2}$ ),

$$
\begin{aligned}
U= & \frac{2\left(2 d+h^{2}\right)^{2} r_{-}^{2} Q^{2}}{\left[2 d-(d-1) h^{2}\right]\left(2 d-d h^{2}\right) \Lambda^{2 d}}\left(\frac{r}{r_{-}}\right)^{2 \frac{2 d-(d-1) h^{2}}{2 d+h^{2}}}\left[1-C_{1}\left(\frac{r}{r_{-}}\right)^{-\frac{2 d-(d-1) h^{2}}{2 d+h^{2}}}\right. \\
& \left.+C_{2}\left(\frac{r}{r_{-}}\right)^{-\frac{4 d-2 d h^{2}}{2 d+h^{2}}}\right], \\
R= & \Lambda\left(\frac{r}{r_{-}}\right)^{\frac{h^{2}}{2 d+h^{2}}}, \\
V= & -\frac{2 Q^{2}}{\left(2-h^{2}\right) \Lambda^{2 d}}\left[2 e^{-2 h \phi}+\frac{h^{2}\left[-2 d+(d+1) h^{2}\right] C_{2}}{\left[2 d-(d-1) h^{2}\right]} e^{-4 \phi / h}\right],
\end{aligned}
$$

In order to make the potential independent from the electric charge, one must choose the integration constant $\Lambda=\left(r_{-} Q\right)^{1 / d}$. As usual, one can introduce a further length scale $L$ in the potential, by performing a rescaling of the variables.

For $C_{1}=C_{2}=0$, the solution is conformal to $(d+2)$-dimensional Lifshitz spacetime. For $h^{2}<2 d /(d-1), C_{2}=0$ and $C_{1}>0$ the solution represents a black brane asymptotical to the conformal Lifshitz spacetime, with a singularity at $r=0$ and a horizon at $r_{h}=C_{1}^{\frac{2 d+h^{2}}{2 d-(d-1) h^{2}}} r_{-}$.

\section{Asymptotically AdS solutions}

In order to derive asymptotically $\mathrm{AdS}_{d+2}$ solutions of our field equations (2.5), we consider again the ansatz (5.1), which expresses the scalar field in terms of a harmonic function $X$ given as in (5.1).

Near the AdS vacuum the scalar field is tachyonic and has mass:

$$
m^{2}=-d / L^{2}
$$

which is always above the BF bound in $d+2$ dimensions. The Riccati equation (2.9) is now solved by:

$$
R=r\left(1-\frac{r_{-}}{r}\right)^{\beta+\frac{1}{2}}, \quad \frac{1}{4}-\beta^{2}=\frac{2}{d} \gamma^{-2}, \quad-\frac{1}{2}<\beta<\frac{1}{2} .
$$

We search again for extremal solutions with $C_{1}=0$. Setting $C_{2}=1 / L^{2}$ in (2.12) and (2.13), we obtain the following asymptotically $\mathrm{AdS}_{d+2}$ domain wall solution and the corresponding potential:

$$
\begin{aligned}
U & =R^{2}=\frac{r^{2}}{L^{2}}\left(1-\frac{r_{-}}{r}\right)^{2 \beta+1}, \quad \gamma \phi=\ln \left(1-\frac{r_{-}}{r}\right) \\
V(\phi) & =-\frac{d}{L^{2}} e^{2 \gamma \beta \phi}\left\{\frac{1}{2}(d+2)\left(1-4 \beta^{2}\right)+\frac{1}{2}\left[4 \beta^{2}(d+2)+d\right] \cosh (\gamma \phi)-2 \beta(d+1) \sinh (\gamma \phi)\right\} .
\end{aligned}
$$


One can easily check that the previous potential satisfies, as expected, $V(0)=-d(d+1) / L^{2}$ and $V^{\prime}(0)=0$. Notice that the metric part of the solutions for the generic case (6.9) is exactly the same as in the $d=2$ case (see Eq. (5.4)). Only the scalar field and the potential are changed. Also in $d+2$ dimensions the potential (6.10) is invariant under the duality transformation (5.10). Dual solutions are easily obtained using (5.10) into Eq. (6.9) and (6.8).

Since the metric functions $U$ and $R$ do not depend on the spacetime dimension, the near-horizon and near-extremal approximate behavior of $U$ and $R$ is the same as in the $d=2$ case. Thus, the hairy extremal solution (6.9) always interpolates between an $\mathrm{AdS}_{d+2}$ vacuum at $r=\infty$ and a domain wall solution (6.3) near $r=r_{-}$.

As in $d=2$, the case $\beta=0$ is particularly simple. The metric part of the solution is still the same as in $d=2$ and is given by Eq. (5.8), whereas the scalar field and the potential are

$$
\phi=\frac{1}{2} \sqrt{\frac{d}{2}} \log \left(1-\frac{r_{-}}{r}\right), \quad V(\phi)=-\frac{d}{L^{2}}\left[\frac{d}{2} \cosh \left(2 \sqrt{\frac{2}{d}} \phi\right)+\frac{d+2}{2}\right] .
$$

For what concerns the existence of nonextremal $C_{1} \neq 0$ solutions in $d+2$ dimensions, and the consequences of the no-hair theorem of Sect. IV the same considerations as in the $d=2$ case hold.

\section{Other solutions}

It is also easy to work out the generalization to $d+2$ dimensions of the model described in subsection $\mathrm{VC}$.

We consider the following ansatz for the scalar field:

$$
a \phi=\log \frac{X_{+}}{X_{-}}, \quad X_{ \pm}=1 \pm\left(\frac{r_{-}}{r}\right)^{n}, \quad a=\sqrt{\frac{8 n^{2}}{d(2 n-1)}} \quad n>\frac{1}{2} .
$$

Near the $\mathrm{AdS}_{d+2}$ vacuum the scalar field is a tachyon with mass

$$
m^{2}=-\frac{n(d+1-n)}{L^{2}},
$$

which is always above the BF bound in $d+2$ dimensions. The PET forbids the existence of black brane solutions for $n \geqslant d+1$, when the square-mass of the scalar becomes positive.

The Riccati equation give the same solution (5.20) as in the $d=2$ case, the metric function $U$ (with $C_{1}=0$ and $C_{2}=\frac{1}{L^{2}}$ ) is given by Eq. (5.21), while the potential becomes

$$
V(\phi)=-\frac{d}{L^{2}}\left(\cosh a \frac{\phi}{2}\right)^{2-\frac{2}{n}}\left[\left(\frac{d+2}{2}-n\right) \cosh a \phi+\left(n+\frac{d}{2}\right)\right] .
$$

Because the metric part of the solution is the same obtained for $d=2$ the near-horizon, nearextremal approximate solution for $U$ and $R$ are identical to those obtained in four dimensions.

\section{SPHERICAL AND HYPERBOLIC SOLUTIONS}

The results of the previous sections can be easily generalized to the case in which the twodimensional sections of the solutions are spherical or hyperbolic. Contrary to the planar case, where it is dimensionless, the metric function $R$, and hence the integration constant $\Lambda$ in the solution (2.14) is now usually taken to have the physical dimension of a length. Therefore, when $\Lambda$ is not determined by the field equation, we shall identify it with the AdS length $L$. 


\section{A. Uncharged black hole solutions}

We first consider the case of four dimensions $(d=2)$. The field equations are given by (2.5), with $\varepsilon= \pm 1$ and the solutions by (2.9)-(2.14).

The generalization of the black brane solutions of sect. IIIA to the case of spherical (or hyperbolic) symmetry is obtained adopting the ansatz (3.1). Substituting the solutions (3.3) with parametrization (3.4), in the general solution of sect. III after rescaling $C_{1}$ and putting $C_{2}=0$, the metric functions take the form

$$
U=\frac{\left(1+h^{2}\right) \varepsilon r_{-}^{2}}{\left(1-h^{2}\right) L^{2}}\left(\frac{r}{r_{-}}\right)^{\frac{2 h^{2}}{1+h^{2}}}\left(1-\frac{C_{1} r_{-}}{r}\right), \quad R=L\left(\frac{r}{r_{-}}\right)^{\frac{1}{1+h^{2}}}
$$

with potential

$$
V=\frac{2 h^{2} \varepsilon}{\left(h^{2}-1\right) L^{2}} e^{-2 \phi / h}
$$

having a simple exponential form, as in the planar case. Notice that we have identified the integration constant $\Lambda$ with the the AdS length $L$.

If $\varepsilon=1$ and $h^{2}<1$, the solutions represent spherically symmetric black holes with conformal Lifshitz asymptotics, exhibiting a singularity at $r=0$, shielded by a horizon at $r_{h}=C_{1} r_{-}$. Solutions exist also for $\varepsilon=-1$ and $h^{2}>1$, they are black holes with conformal Lifshitz asymptotics and horizons with hyperbolic topology.

\section{B. Charged black hole solutions}

We now try to extend the previous solutions to the case of nonvanishing electric charge, generalizing those of sect. IIIB With the parametrization (3.7), the solution reads, after a redefinition of the constants $C_{1}$ and $C_{2}$,

$$
\begin{aligned}
U= & \frac{\left(4+h^{2}\right) r_{-}^{2}}{4-h^{2}}\left[-\frac{\varepsilon}{\Lambda^{2}}\left(\frac{r}{r_{-}}\right)^{\frac{8}{4+h^{2}}}+\frac{\left(4+h^{2}\right) Q^{2}}{\left(2-h^{2}\right) \Lambda^{4}}\left(\frac{r}{r_{-}}\right)^{2 \frac{4-h^{2}}{4+h^{2}}}+C_{2}\left(\frac{r}{r_{-}}\right)^{\frac{2 h^{2}}{4+h^{2}}}\right. \\
& \left.-C_{1}\left(\frac{r}{r_{-}}\right)^{\frac{4-h^{2}}{4+h^{2}}}\right], \quad R=\Lambda\left(\frac{r}{r_{-}}\right)^{\frac{h^{2}}{4+h^{2}}},
\end{aligned}
$$

with

$$
V=-\frac{4 Q^{2}}{\left(2-h^{2}\right) \Lambda^{4}} e^{-2 h \phi}+\frac{8 \varepsilon}{\left(4-h^{2}\right) \Lambda^{2}} e^{-h \phi}+\frac{2 h^{2}\left(4-3 h^{2}\right) C_{2}}{\left(4+h^{2}\right)\left(4-h^{2}\right)} e^{-4 \phi / h} .
$$

Contrary to the planar case, if $\varepsilon \neq 0$, one cannot eliminate from the potential the dependence on $Q$ by a suitable choice of the integration constants: one ought in fact to impose $\Lambda^{2}=Q=1$. However, the solution with $Q=0, C_{2} \neq 0$ may still have interest. For $\varepsilon>0, h^{2}>4$ and $C_{1}>0$, such solution represents a black hole with domain wall asymptotical behavior, a singularity at $r=0$ and one or two horizons, depending on the value of $C_{2}$. The asymptotic behavior is dictated by the $C_{2}$ term. The potential is the sum of two exponential.

\section{Asymptotically AdS solutions}

In this section, we wish to generalize the asymptotically anti-de Sitter solutions obtained using the ansatz (5.1), in the case $\varepsilon \neq 0$. The solution (5.2) for the radial function still holds, while, after 
the usual rescaling of $C_{2}$, the metric function $U$ becomes in the special case $C_{1}=0$, corresponding to an extremal black hole,

$$
U=-\frac{\varepsilon r^{2}}{2 \beta(1+4 \beta) L^{2}} X^{-2 \beta}\left[1-(1+4 \beta) \frac{r_{-}}{r}\right]+\frac{C_{2} r^{2}}{L^{2}} X^{2 \beta+1},
$$

where we have rescaled $C_{2}$, set $L=\Lambda r_{-}$, and $-1 / 4<\beta<0$. The potential is then

$$
V(\phi)=-\frac{\varepsilon}{2 \beta(1+4 \beta)} V_{1}(-\gamma, \phi)+C_{2} V_{1}(\gamma, \phi) .
$$

where $V_{1}(\gamma, \phi)$ is given by Eq. (5.5) . The metric is singular at $r=0$, while, when $C_{2} \neq 0$, in general the solution is a black hole, whose horizon structure cannot be determined analytically. As in the planar case, solutions with $C_{1} \neq 0$ exist, but it is not possible to eliminate $C_{1}$ from the potential. Hence if a family of black hole solutions exists for the potential (7.6), it must be determined numerically.

An interesting property of the potential (7.6) is the symmetry between its two terms for $\phi \rightarrow-\phi$. In particular, choosing $C_{2}=\varepsilon / 2 \beta(1+4 \beta) L^{2}$, the potential becomes

$$
\begin{aligned}
V(\phi)= & -\frac{2 \varepsilon}{\beta(1+4 \beta) L^{2}}\left\{\left(\beta+\frac{1}{2}\right)(4 \beta+1) \sinh \left[2\left(\beta-\frac{1}{2}\right) \gamma \phi\right]-\left(8 \beta^{2}-2\right) \sinh [2 \beta \gamma \phi]\right. \\
& \left.+\left(\beta-\frac{1}{2}\right)(4 \beta-1) \sinh [(2 \beta+1) \gamma \phi]\right\} .
\end{aligned}
$$

The most interesting case is $C_{2}=0$ and $\varepsilon=1$. With this assumption, using the invariance of the field equations under the rescaling $R \rightarrow \frac{1}{\lambda} R, U \rightarrow \lambda^{2} U, V \rightarrow \lambda^{2} V$ and changing the sign of $\gamma$ in Eq. (5.1), the potential (7.6) can be brought into the form $V=V_{1}(\gamma, \phi)$ where, as usual, $V_{1}(\gamma, \phi)$ is given by Eq. (5.5). Solution (7.5) becomes

$$
U=-\frac{r^{2}}{L^{2}} X^{-2 \beta}\left[1-(1+4 \beta) \frac{r_{-}}{r}\right], \quad R=\frac{L r}{r_{-} \sqrt{-2 \beta(1+4 \beta)}} X^{\beta+\frac{1}{2}}, \quad \gamma \phi=-\log X .
$$

In the range of definition, $-1 / 4<\beta<0$, the solution has no horizon. However, as we will see when we consider the near-horizon, near extremal solution, it cannot be considered an extremal black hole.

We conclude by observing that a solution can be found also in the particular case $\beta=0, \gamma=2$. In this case,

$$
\phi=\frac{1}{2} \log X, \quad R=\Lambda r X^{1 / 2},
$$

and then

$$
\begin{aligned}
U & =\frac{2 \varepsilon r\left(r-r_{-}\right)}{L^{2}}\left[\frac{r_{-}}{r-r_{-}}+\log \left(\frac{r-r_{-}}{r}\right)\right], \\
V & =-\frac{4 \varepsilon}{L^{2}}[4 \phi+2 \phi \cosh (2 \phi)-3 \sinh (2 \phi)] .
\end{aligned}
$$

\section{Charged asymptotically AdS solutions}

We consider now the solutions of the previous section with $Q \neq 0$, but $C_{2}=0$. The only change is in the function $U$,

$$
\begin{aligned}
U= & -\frac{\varepsilon r^{2}}{2 \beta(1+4 \beta) L^{2}} X^{-2 \beta}\left[1-(1+4 \beta) \frac{r_{-}}{r}\right] \\
& +\frac{8 \mu r^{2}}{3\left(1-36 \beta^{2}\right)} X^{-4 \beta}\left[1+(1+6 \beta) \frac{r_{-}}{r}+3 \beta(1+6 \beta) \frac{r_{-}^{2}}{r^{2}}\right],
\end{aligned}
$$


and in the potential

$$
V(\phi)=-\frac{\varepsilon}{2 \beta(1+4 \beta)} V_{1}(-\gamma, \phi)-\frac{2 \mu L^{2}}{3 \beta(1+4 \beta)} V_{2}(\gamma, \phi),
$$

where we have defined $L=\Lambda r_{-}, \mu=\frac{Q^{2}}{r^{3} \Lambda^{4}}$ and $V_{1}(\gamma, \phi)$ and $V_{2}(\gamma, \phi)$ are given respectively by Eqs. (5.5) and (5.15). Therefore $\mu, L$ and $\beta$ are parameters of the action, $Q$ is a free parameter and $r_{-}=\mu L^{4} / Q^{2}$. Multiple horizons may occur, but cannot be determined analytically for generic $\beta$.

\section{E. Spherical solutions generated from the planar ones}

For $d=2$, spherical solutions can simply be generated from the planar ones just by exploiting the fact that the field equations (2.5) are linear in the metric function $U$. This method permits to find spherical solutions for a given form of the potential. This fact may be very useful when one wants to compare planar and spherical solutions of the same model or when the method described in the previous subsection gives a singular result (e.g. $\beta=0$ in Eq. (7.5)).

Indicating with $U_{0}, R_{0}=r X^{\beta+1 / 2}, \phi_{0}=\gamma^{-1} \ln X$, where $X$ is the harmonic function (5.1), a solution of the field equations (2.5) for $\varepsilon=0$ and $d=2$, it follows from the linearity in $U$ of the field equations that a solution of (2.5) for $\varepsilon= \pm 1$ and $d=2$ is given by

$$
U=U_{0}+\varepsilon X^{-2 \beta}, \quad R=R_{0}, \quad \phi=\phi_{0} .
$$

This method can be used to generate $\varepsilon= \pm 1$ solutions for the potentials (5.5) and (5.15) from the planar solutions respectively given by (5.4) and (5.14). In the uncharged case, i.e for the potential (5.5) we have

$$
U=X^{2 \beta+1} \frac{r^{2}}{L^{2}}+\varepsilon X^{-2 \beta}, \quad R=r X^{\beta+\frac{1}{2}}, \quad \gamma \phi=\ln X, \quad X=1-\frac{r_{-}}{r}, \quad \frac{1}{4}-\beta^{2}=\gamma^{-2} .
$$

Notice that, differently from Eqs. (7.7) this solution holds in the full range $-1 / 2<\beta<1 / 2$ of the parameter $\beta$. For $\beta=0$ we get the spherical extremal solution of the model (5.7):

$$
U=\frac{r^{2}}{L^{2}}-\frac{r r_{-}}{L^{2}}+\varepsilon, \quad R=r X^{1 / 2}, \quad \phi=\frac{1}{2} \ln X .
$$

One can easily check that solution (7.10) is an extremal $T=0$ solution, in fact the zeros of $U$ are always behind the origin of the radial coordinate at $r=r_{-}$.

Eqs. (7.7) and (7.10) are solutions of the same model with potential $V_{1}(\gamma, \phi)$ given by (5.5). Because $V_{1}(\gamma, \phi)$ is invariant under the duality transformation (5.10), one can generate from (77.7), (17.10) two other solutions of the same model just by reversing the sign of $\beta$ and $\phi$.

\section{F. Near-horizon, near-extremal solution with spherical topology}

Let us now consider the near-horizon approximation of the solutions (7.7) and (7.10). One can obtain the near-horizon, near-extremal solution by first shifting $r \rightarrow r+r_{-}$and expanding near $r=0$. Then one solves the field equations perturbatively near $r=0$ using the the extremal, near-horizon solution as zero-th order approximation.

In the case of solution (7.7) this procedure gives the solution

$$
\begin{aligned}
& U=\left(\frac{r_{-}}{L}\right)^{2} x^{-2 \beta}[(1-4 \beta(1+2 \beta)) x-C], \\
& R=\frac{L x^{\beta+\frac{1}{2}}}{\sqrt{-2 \beta(1+4 \beta)}}\left[1-\frac{2}{C} \beta(2 \beta-1) x\right], \quad \gamma \phi=-\ln x+\frac{4 \beta}{C} x,
\end{aligned}
$$


where $C$ is an integration constant and $x=r / r_{-}$. For $C>0$ the solutions describe black holes with a regular horizon at $x=x_{h}=C /(1-4 \beta(1+2 \beta))$ with $R\left(x_{h}\right) \neq 0$ and a singularity at $r=0$. The Hawking temperature of the horizon is $T=(1 / 4 \pi)\left(r_{-}^{2} / L^{2}\right) x_{h}{ }^{-2 \beta}$. The solution (7.12) is singular for $C=0$, although $T \rightarrow 0$ as $C \rightarrow 0$. For $C<0$ we have solutions with no horizon and in particular for $C=C_{0}=4 \beta$ we get the solution (7.7) in the near-horizon approximation.

Because solution (17.12) has a singularity at $r=0$ (corresponding to the singularity of (7.7) at $r=r_{-}$), one should reject solutions with $C<0$ as unphysical. This gives a strong hint about the nature of our solution (7.7): it is an isolated solution disconnected from the continuous part of the black hole spectrum at $C>0$ by solutions with naked singularities.

In the case of solution (7.10), near-horizon, near-extremal solutions are given by

$$
\begin{aligned}
& U=\frac{r_{-}^{2}}{L^{2}}\left(x^{2 \beta+1}-C x^{-2 \beta}\right)+2 \beta x^{-2 \beta+1}, \\
& R=r_{-} x^{\beta+\frac{1}{2}}\left(1+\frac{L^{2}}{C r_{-}^{2}}\left(\beta-\frac{1}{2}\right) x\right), \quad \gamma \phi=\ln x+\frac{L^{2}}{C r_{-}^{2}} x,
\end{aligned}
$$

where $C$ is an integration constant. For $C>0$ the solutions have a regular horizon, which at leading order is located at $x=x_{h}=C^{1 /(4 \beta+1)}$ when $C$ is small, $C<\left(r_{-}^{2} /\left(L^{2}(1-2 \beta)\right)\right)^{-(4 \beta+1) /(4 \beta)}$. This is consistent with a near-extremal approximation. The Hawking temperature of the horizon is $T=(1 / 4 \pi)\left(r_{-}^{2} / L^{2}\right)(4 \beta+1) x_{h}{ }^{2 \beta}$. The solution (7.13) is singular for $C=0$. For $C<0$ we have solutions with no horizon and, in particular, for $C=C_{0}=L^{2} / r_{-}^{2}$ we get solution (7.10) in the near-horizon approximation.

Also solution (7.13) has singularity at $r=0$, which corresponds to the singularity of (7.10) at $r=r_{-}$. Hence, solutions with $C<0$ have naked singularities and solution (7.10) is disconnected from the continuous part of the black hole spectrum at $C>0$.

\section{CONCLUSIONS}

In this paper, we have presented a general method for finding static, radially symmetric, analytic solutions of Einstein and Einstein-Maxwell gravity minimally coupled to a scalar field. Rather than assuming a particular form of the scalar self-interaction potential, our method starts from an ansatz for the scalar field profile and determines, together with the metric functions, the corresponding form of the potential. For this reason it is particularly suitable for applications to the AdS/CFT correspondence. We have investigated in detail two related applications of our method.

We have first derived a new no-hair theorem about the existence of black hole solutions of Einstein gravity with scalar hair. As a second application, we have derived broad classes of exact analytic hairy solutions of Einstein and Einstein-Maxwell gravity minimally coupled to a scalar. These solutions have been derived using rather general and simple ansätze for the scalar (in terms of harmonic and logarithmic functions). They cover many different situations: four or higher dimensions; solutions with planar, spherical or hyperbolic horizon topology; solutions with AdS, domain-wall and conformal Lifshitz asymptotics; solutions interpolating between an AdS spacetime in the asymptotic region and domain-wall or conformally Lifshitz behaviour in the near-horizon region. Also the class of potentials for the scalar field characterizing these models are broad ranging from the simple exponential potential -known to give rise in many situations to domain wall and Lifshitz solutions [20, 24, 25, 29] - to more general forms, that contain as a particular case $\mathcal{N}=8$ gauged supergravity in $4 \mathrm{D}$ truncated to the $U(1)$ sector.

Our investigation has shown that Einstein gravity minimally coupled to a scalar field has a rich spectrum of solutions with non trivial scalar hair and AdS asymptotics, which may play an important role in applications of the AdS/CFT correspondence to condensed matter and strongly coupled QFTs.

Our approach has a main drawback. In some situations it does not allow to find a full oneparameter family of black holes, i.e. the full spectrum of solutions for different temperatures, but 
only 'extremal' $T=0$ solutions. Moreover, what we have called extremal solutions always present a curvature singularity at $r=0$.

Although our method always allows to find one-parameter families of near-horizon near-extremal solutions, interpolating solutions with AdS asymptotics can be found only in the extremal case. Moreover, in many situations, it is not even clear whether or not such solutions exist. This is a particularly important question in the cases in which the exact solution interpolates between the AdS spacetime and a near-horizon domain wall or Lifshitz spacetime. In the spherical case we have found strong evidence that our exact solutions represent isolated solutions disconnected from the continuous part of the spectrum. A final answer to these question involves numerical computation. We will address these and other issues concerning the solutions found in this work - in particular a detailed discussion of the thermodynamics and of the causal structure of the solutions and a precise characterization of the spacetime singularities - in a subsequent paper.

\section{Acknowledgments}

We thank M. Melis and P. Pani for discussions and illuminating comments. This work was partially supported by a grant funded by P.O.R. SARDEGNA F.S.E. 2007-2013.

[1] W. Israel, Phys. Rev. 164, 1776 (1967).

[2] J. D. Bekenstein, Phys. Rev. D51, 6608 (1995).

[3] G. Gibbons and K.-i. Maeda, Nucl.Phys. B298, 741 (1988).

[4] D. Garfinkle, G. T. Horowitz, and A. Strominger, Phys. Rev. D43, 3140 (1991).

[5] M. Cadoni and S. Mignemi, Phys. Rev. D 48, 5536 (1993), 9305107.

[6] S. Monni and M. Cadoni, Nucl. Phys. B466, 101 (1996), hep-th/9511067.

[7] A. E. Mayo and J. D. Bekenstein, Phys. Rev. D54, 5059 (1996), gr-qc/9602057.

[8] J. M. Maldacena, Adv. Theor. Math. Phys. 2, 231 (1998), hep-th/9711200.

[9] G. T. Horowitz and J. Polchinski (2006), gr-qc/0602037.

[10] S. Sachdev and M. Mueller (2008), 0810.3005.

[11] S. A. Hartnoll, C. P. Herzog, and G. T. Horowitz, Phys. Rev. Lett. 101, 031601 (2008), 0803.3295.

[12] S. A. Hartnoll, C. P. Herzog, and G. T. Horowitz, JHEP 12, 015 (2008), 0810.1563.

[13] C. P. Herzog, J. Phys. A42, 343001 (2009), 0904.1975.

[14] S. A. Hartnoll (2009), 0903.3246.

[15] T. Torii, K. Maeda, and M. Narita, Phys.Rev. D64, 044007 (2001).

[16] T. Hertog, Phys. Rev. D74, 084008 (2006), gr-qc/0608075.

[17] P. Breitenlohner and D. Z. Freedman, Phys. Lett. B115, 197 (1982).

[18] K. Skenderis, Class.Quant.Grav. 19, 5849 (2002), hep-th/0209067.

[19] G. T. Horowitz and M. M. Roberts, Phys.Rev. D78, 126008 (2008), 0810.1077.

[20] M. Cadoni, G. D'Appollonio, and P. Pani, JHEP 03, 100 (2010), 0912.3520.

[21] G. T. Horowitz (2010), 1002.1722.

[22] M. Cadoni and P. Pani, JHEP 1104, 049 (2011), 1102.3820.

[23] M. Ammon, J. Erdmenger, M. Kaminski, and P. Kerner, Phys. Lett. B680, 516 (2009), 0810.2316.

[24] C. Charmousis, B. Gouteraux, and J. Soda, Phys. Rev. D80, 024028 (2009), 0905.3337.

[25] K. Goldstein, S. Kachru, S. Prakash, and S. P. Trivedi (2009), 0911.3586.

[26] C.-M. Chen and D.-W. Pang, JHEP 1006, 093 (2010), 1003.5064.

[27] K. Goldstein, N. Iizuka, S. Kachru, S. Prakash, S. P. Trivedi, et al., JHEP 1010, 027 (2010), 1007.2490.

[28] Y. Liu and Y.-W. Sun, JHEP 07, 099 (2010), 1006.2726.

[29] E. Perlmutter, JHEP 02, 013 (2011), 1006.2124.

[30] S. Kachru, X. Liu, and M. Mulligan, Phys. Rev. D78, 106005 (2008), 0808.1725.

[31] G. Bertoldi, B. A. Burrington, and A. W. Peet, Phys.Rev. D80, 126004 (2009), 0907.4755.

[32] G. Bertoldi, B. A. Burrington, and A. Peet, Phys.Rev. D80, 126003 (2009), 0905.3183.

[33] M. Dehghani, R. Mann, and R. Pourhasan (2011), 1102.0578.

[34] G. Bertoldi, B. A. Burrington, A. W. Peet, and I. G. Zadeh (2011), 1101.1980.

[35] C. Charmousis, B. Gouteraux, B. S. Kim, E. Kiritsis, and R. Meyer, JHEP 11, 151 (2010), 1005.4690. 
[36] B. Gouteraux and E. Kiritsis (2011), 1107.2116.

[37] M. J. Duff and J. T. Liu, Nucl. Phys. B554, 237 (1999), hep-th/9901149.

[38] H. Lu, J.-w. Mei, C. N. Pope, and J. F. Vazquez-Poritz, Phys. Lett. B673, 77 (2009), 0901.1677.

[39] C. Martinez, R. Troncoso, and J. Zanelli, Phys.Rev. D70, 084035 (2004), hep-th/0406111.

[40] R.-G. Cai and Y.-Z. Zhang, Phys. Rev. D54, 4891 (1996), gr-qc/9609065.

[41] R.-G. Cai, J.-Y. Ji, and K.-S. Soh, Phys. Rev. D57, 6547 (1998), gr-qc/9708063.

[42] R.-G. Cai and A. Wang, Phys. Rev. D70, 084042 (2004), hep-th/0406040.

[43] C. J. Gao and S. N. Zhang, Phys.Rev. D70, 124019 (2004), hep-th/0411104.

[44] S. Mignemi (2009), 0907.0422.

[45] I. Kanitscheider and K. Skenderis, JHEP 0904, 062 (2009), 0901.1487.

[46] H. Boonstra, K. Skenderis, and P. Townsend, JHEP 9901, 003 (1999), hep-th/9807137.

[47] S. Mignemi and D. Wiltshire, Class.Quant.Grav. 6, 987 (1989).

[48] M. Cadoni, S. Mignemi, and M. Serra, In preparation (2011).

[49] T. Hertog and K. Maeda, JHEP 0407, 051 (2004), hep-th/0404261. 